\title{
Subsystem Throughputs of a Clinical Picture Archiving and Communications System
}

\author{
Albert W.K. Wong and H.K. Huang
}

\begin{abstract}
We measured the throughtput rates of individual picture archiving and communications system (PACS) subsystems including the acquisition, archive, display, and communication network as a basis of evaluation the overall throughput of our clinical PACS. The throughput rate of each PACS subsystem was measured in terms of average residence time of individual images in the subsystem. The residence time of an image in a PACS subsystem was determined by the total time the image was required to be processed within the subsystem. The overall throughput of the PACS was measured as the total residence time of an image in the various subsystems. We also measured throughputs of the PACS subsystems using three types of networks (Ethernet; fiber distributed data interface; and UltraNet, UltraNetwork Technologies, San Jose, CA), and the results were compared. Approximately $\mathbf{2 0 0}$ gigabytes of data transactions including magnetic resonance, computed tomography and computed radiography images from our PACS were analyzed. Results showed that PACS throughput was limited by three major factors: (1) low-speed data interface used in the radiologic imaging devices and archive devices; (2) competition for systems processing time among the PACS processes; and (3) network degradation caused by heavy network traffic. We concluded that PACS performance could be improved with a well-designed network architecture, $a$ job prioritizing mechanism, and an image routing strategy. However, device-dependent low-speed data interface has limited PACS performance.
\end{abstract}

KEY WORDS: picture archiving and communication system (PACS), PACS controller, PACS subsystem, computed radiography (CR), image residence time.

A PICTURE ARCHIVING and communication system (PACS) comprises four major

From the Department of Radiological Sciences, Center for Health Sciences, AR-277, UCLA School of Medicine, Los Angeles, CA 90024-1721.

Supported in part by the National Cancer Institute Public Health Service grant no POI CA 51198 and Eastman Kodak Company (Rochester, NY).

Address reprint requests to Albert W.K. Wong, BS, Department of Radiology, Medical Center, University of California, San Francisco, PO Box 628, 521 Parnassus, San Francisco, CA 94143-0840.

Reprinted with permission from Medical Imaging VI: Picture Archiving and Communications Systems, Society of PhotoOptical Instrumentation Engineers, 1992.

Copyright 1992 by The Society of Photo-Optical Instrumentation Engineers

0-8194-0806-9/92/\$4.00 imaging components: acquisition, archiving, display, and communication. Because of their diverse functions, each of these components is a subsystem in the PACS that operates independently and communicates simultaneously with other components creating an integrated system. Hence, any delay of one of these components in processing its images will cause an impact to the overall throughput of the entire PACS. When a PACS is in clinical operation, servicing the radiologists and referring physicians is its most important function. The timeliness of delivering an image to a display station or print station on completion of acquiring the image from a radiologic imaging device or retrieving the image from an optical library determines the reliability and acceptance of a PACS. Therefore, the strategy we implemented in the PACS at the University of California at Los Angeles (UCLA) is used to minimize the image delivery time. To achieve this we prioritized job control and used a queuing mechanism to manage all of the PACS processes running on individual PACS computers so that a display station or print station always had the highest priority of receiving its service.

When successful installation of a fiber-optic communication network connecting the Center for Health Sciences (inpatient facility), the Medical Plaza (outpatient facility), and the Advanced Magnetic Resonance (MR) Imaging Center (2.0 km off-campus) in October $1990,^{1-3}$ we began implementing a large-scale PACS in the radiology department. ${ }^{4,5}$ Individual PACS modules that have been implemented earlier in the department ${ }^{6-8}$ were connected to the multiple image communication networks. This PACS is characterized by its centralized image management, multiple networking, and distributed functionality. All images acquired from the $3 \mathrm{MR}$ scanners, 4 computed-tomography (CT) scanners, 3 computed-radiography (CR) units, and 1 laser film digitizer are sent from their acquisition computers via the Ethernet or FDDI (fiber distributed data interface) networks to the PACS controllers, where these images are archived to optical disks and distributed over the high- 
speed UltraNet network (Ultra Network Technologies, San Jose, CA) to remote display stations. By using a centralized image management system, this department-wide PACS facilitates its ability to providing intersectional referencing throughout the entire radiology department.

Figure 1 and Fig 2 are the block diagrams showing the configuration of the UCLA PACS at the Center for Health Sciences, Medical Plaza, and Advanced MR Imaging Center. At each site of the Center for Health Sciences and the Medical Plaza, a central node comprising a PACS controller (Sun SPARCserver 490; Sun Microsystems, Mountain View, CA), a database server (Sun SPARCserver 490), a Kodak ADL 6800 optical library (Eastman Kodak Company, Rochester, NY), and a Kodak standalone optical drive manages all the images acquired from the respective site. These two central nodes are interconnected with the rest of the PACS computers via the multiple communication networks. In their normal operation, the two central nodes run concurrently but operate independently. However, if any component in a central node fails to operate, its mirrored equipment in the second central node will automatically reconfigure itself instantaneously and perform the duties of both devices so that no delay in image receiving, archiving, or routing should occur.

The distributed PACS has been in clinical service since January, 1992. Currently, it acquires 2.0 Gbytes of image data every workday. This report describes the performance of the individual PACS subsystems. The results were analyzed from 200 Gbytes of data collected in the last 4 months. These performance measurements were conducted to identify the major factors that degrade the performance of a PACS and to establish new strategies so that fast service with minimal delay is provided to the clinic.

\section{MATERIALS AND METHODS}

The performance measurement was conducted in a real time clinical environment from our departmental PACS on

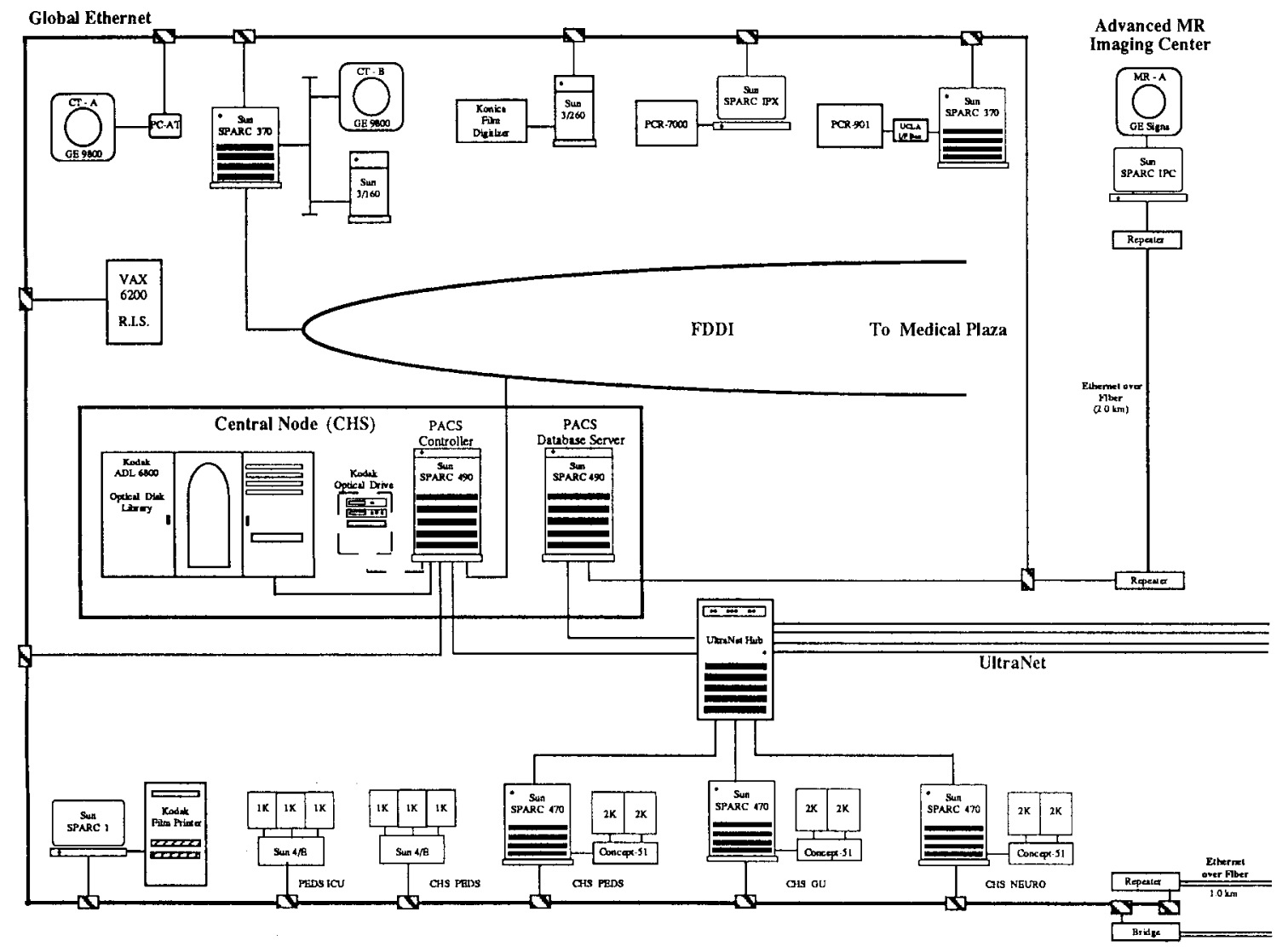

Fig 1. UCLA PACS network: Center for Health Sciences and Advanced MR Imaging Center (as of April 31, 1992). 


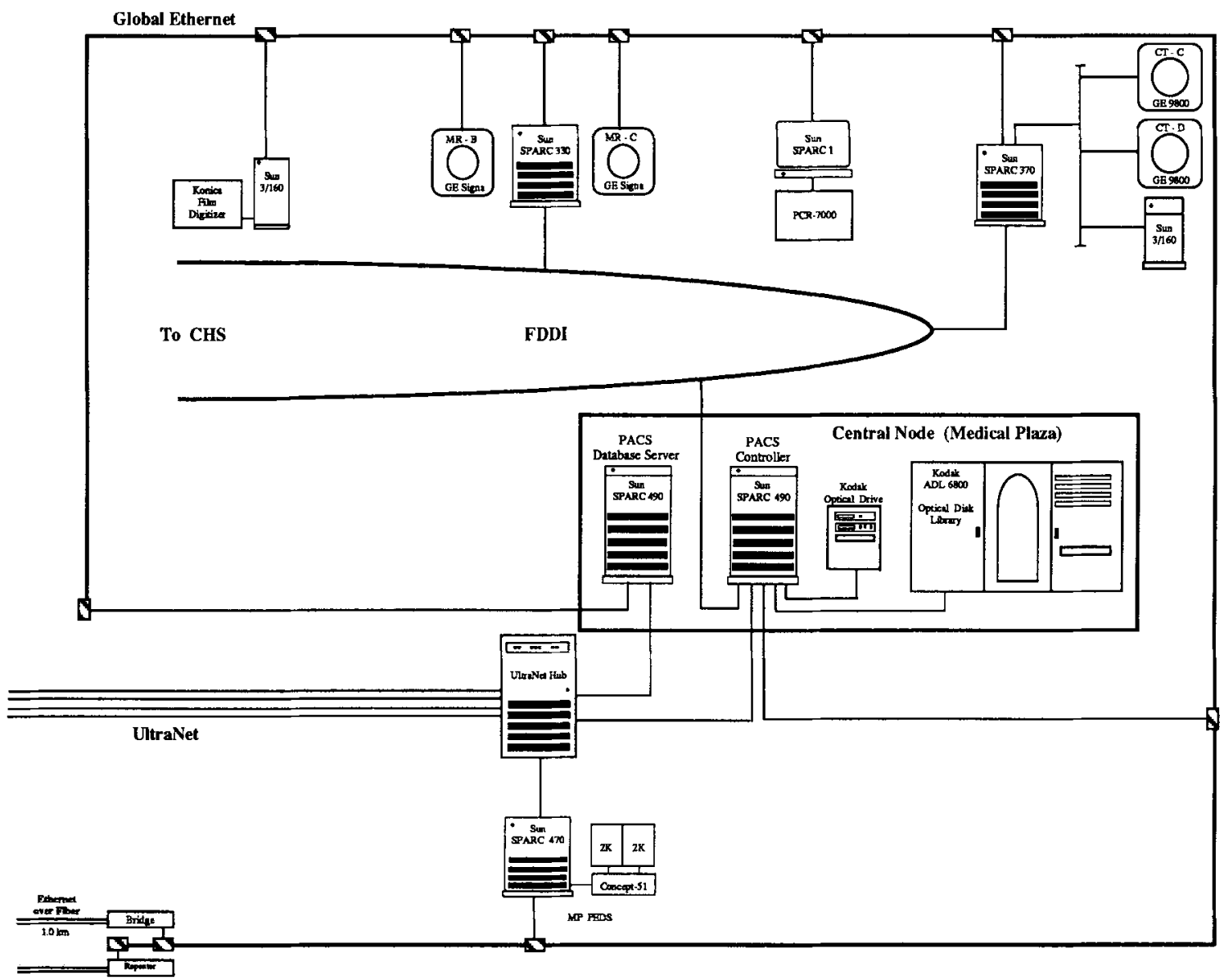

Fig 2. UCLA PACS network: Medical Plaza (as of April 31, 1992).

a 24-hour per day, 7-day per week basis. Approximately 200 Gbytes of transaction data including MR, CT, and CR images were analyzed. Equipment encountered in the performance measurements included $3 \mathrm{MR}$ scanners, $4 \mathrm{CT}$ scanners, 3 CR units, 2 optical disk libraries, 6 display units (four $2 \mathrm{~K}[2,048 \times 2,048$ pixels $]$ and two $1 \mathrm{~K}[1,024 \times 1,024$ pixels]), 3 types of networks, and the corresponding host computers. Tables 1 through 4 list the equipment and their host computers that comprised the acquisition, archiving, display, and communication network subsystems.

We measured the throughput of a PACS subsystem in terms of the average residence time of individual images in that subsystem. The residence time of an image in a PACS subsystem is defined as the total time required to process the image to accomplish a particular task within that subsystem. The overall throughput of a PACS was then measured by the total residence time of an image in the various subsystems.

Each of the PACS subsystems may perform several tasks, and each task may be accomplished by several processes. An archive subsystem, for example, performs three major tasks: image archival, image retrieval, and image routing. To perform the image retrieval task, a server process accepts retrieval requests from the display stations, a retrieve process retrieves images from the optical disks, and a send process sends images to the destination display stations. These three processes communicate with each other through a queuing mechanism and run cooperatively to accomplish the same task. In this example, the retrieval residence time of an image in the archive subsystem was measured by the

Table 1. Components of the Acquisition Subsystem

\begin{tabular}{clcll}
\hline Modality & Model & Quantity & Host Computer & Data Interface \\
\hline MR & GE Signa & 3 & Sun SPARC 330 or Sun SPARC IPC & FTP via Ethernet \\
CT & GE CT-9800 & 4 & Sun SPARC 370 & GE Genesis via Ethernet \\
CR & PCR-901 & 1 & Sun SPARC 370 & UCLA interface box via Ethernet \\
& PCR-7000 & 2 & Sun SPARC IPX or Sun SPARC 1 & Philips PIP via Ethernet \\
\hline
\end{tabular}


Table 2. Components of the Archive Subsystem

\begin{tabular}{ccc}
\hline Host Computer & Optical Library & Database \\
\hline & $\begin{array}{c}\text { 680-Gbyte Kodak ADL 6800 } \\
\text { with SCSI data interface }\end{array}$ & Sybase \\
\hline
\end{tabular}

elapsed time an archive server received the retrieve request, retrieved the image from an optical disk, and finished sending the image to the destination display station.

Following this definition, we measured the various image residence times from the acquisition, archive, display, and communication network subsystems according to the particular tasks each of these subsystems performed. In our experiments, the following image residence times were measured: acquisition residence time, archival residence time, retrieval residence time, distribution residence time, display residence time, and network residence time. Table 5 defines these image residence times and Fig 3 shows the PACS processes that were encountered in the measurement of these image residence times.

\section{RESULTS}

The image residence times we measured from the individual PACS subsystems represented the required processing times in a clinical environment on images within the corresponding subsystems. Compared with their point-to-point data rates (see below), these image residence times individually showed a considerable low throughput capacity caused by factors such as multitasking, network traffic, or device-todevice data communication. A description of the point-to-point data rates and the achieved throughputs measured from each of the PACS subsystems follows.

\section{Acquisition Residence Time}

An acquisition subsystem performed three major tasks: (1) acquiring image data and patient demographic information from radiologic imaging devices, (2) converting image data and patient demographic information to the UCLA PACS format, and (3) sending reformatted image files to a PACS controller. Images from various modalities were acquired via different data interface. The current technology adopted by major manufacturers limited the transfer speed of image data from a radiologic imaging
Table 4. Components of the Network Subsystem

\begin{tabular}{lcc}
\hline Network & Transmission Media & $\begin{array}{c}\text { Specified Bandwidth } \\
\text { (Megabits/s) }\end{array}$ \\
\hline Ethernet & Coaxial cable & 10 \\
FDDI & Optical fiber & 100 \\
UltraNet & Optical fiber & 1000 \\
\hline
\end{tabular}

device to its acquisition computer. ${ }^{9,10}$ This constituted the major factor that affected the overall throughput of a PACS. The real time acquisition residence times of the $\mathrm{MR}, \mathrm{CT}$, and $\mathrm{CR}$ images are described below.

MR image acquisition. The GE Signa scanners (General Electric Medical Systems, Milwaukee, WI) transmitted images at a data rate of $95 \mathrm{kbytes} / \mathrm{s}$ via the Ethernet using standard file transfer protocol (FTP). The acquisition computers scanned the image directories within the scanners periodically and extracted new images from the scanners. However, there was no information from the scanners to indicate the completion of a scan. An acquisition process knew that a scan was complete only when a slice image from a new scan was detected or the number of slice images remained constant over a certain period of time. This caused a significant delay in acquiring a complete sequence of slice images to our PACS. (All slice images from one scan are defined as a complete image sequence and are packed into a PACS image file through the reformat process.) Reformatting slice images from one scan into a PACS image file was done during the waiting time of receiving the next slice image and therefore the acquisition time of an image sequence overlapped the reformat time of its individual slice images. The reformatted images were sent at an average size of 1.96 Mbytes to the PACS controllers via the FDDI or the Ethernet network.

CT image acquisition. The GE CT-9800 scanners (General Electric Medical Systems) used Genesis data interface to transmit images at a data rate of $75 \mathrm{kbytes} / \mathrm{s}$. (Genesis is a prototype data interface developed for UCLA by General Electric as a transition of our future adoption of

Table 3. Components of the Display Subsystem

\begin{tabular}{llll}
\hline & Host Computer & \multicolumn{1}{c}{ Local Storage } & Display Unit \\
\hline $2 \mathrm{~K}$ & Sun SPARC 470 & 2.6 -Gbyte Concept-51 ptd & MegaScan display system with two portrait-mode monitors \\
$1 \mathrm{~K}$ & Sun 4/E & 1.3 -Gbyte Sun magnetic disk & UCLA display controller with three portrait-mode monitors \\
\hline
\end{tabular}


Table 5. Definition of Acquisition, Archival, Retrieval, Distribution, Display, and Network Residence Times

\begin{tabular}{|c|c|c|}
\hline $\begin{array}{l}\text { Image } \\
\text { Residence Time }\end{array}$ & $\begin{array}{l}\text { Subsystem Where } \\
\text { Measurement } \\
\text { Performed }\end{array}$ & Definition \\
\hline Acquisition & Acquisition & $\begin{array}{l}\text { Total time of receiving an image from a radiologic imaging device, reformatting } \\
\text { the image, and sending the image to a PACS controller. }\end{array}$ \\
\hline Archival & Archive & $\begin{array}{l}\text { Total time of receiving an image from an acquisition node, updating the PACS da- } \\
\text { tabase, and archiving the image to an optical disk. }\end{array}$ \\
\hline Retrieval & Archive & $\begin{array}{l}\text { Total time of retrieving an image from an optical disk and sending the image to a } \\
\text { display station. }\end{array}$ \\
\hline Distribution & Archive & $\begin{array}{l}\text { Total time of receiving an image from an acquisition node, updating the PACS da- } \\
\text { tabase, and sending the image to a display station. }\end{array}$ \\
\hline Display (2K) & Display & $\begin{array}{l}\text { Total time of receiving an image from a PACS controller, transferring the image } \\
\text { from the Sun disk to the ptd, and displaying the image in a } 2 \mathrm{~K} \text { monitor. }\end{array}$ \\
\hline Display (1K) & Display & $\begin{array}{l}\text { Total time of receiving an image from a PACS controller and displaying the image } \\
\text { in a } 1 \mathrm{~K} \text { monitor. }\end{array}$ \\
\hline Network & Network & $\begin{array}{l}\text { Total travelling time of an image from one PACS component to another via a net- } \\
\text { work. }\end{array}$ \\
\hline
\end{tabular}

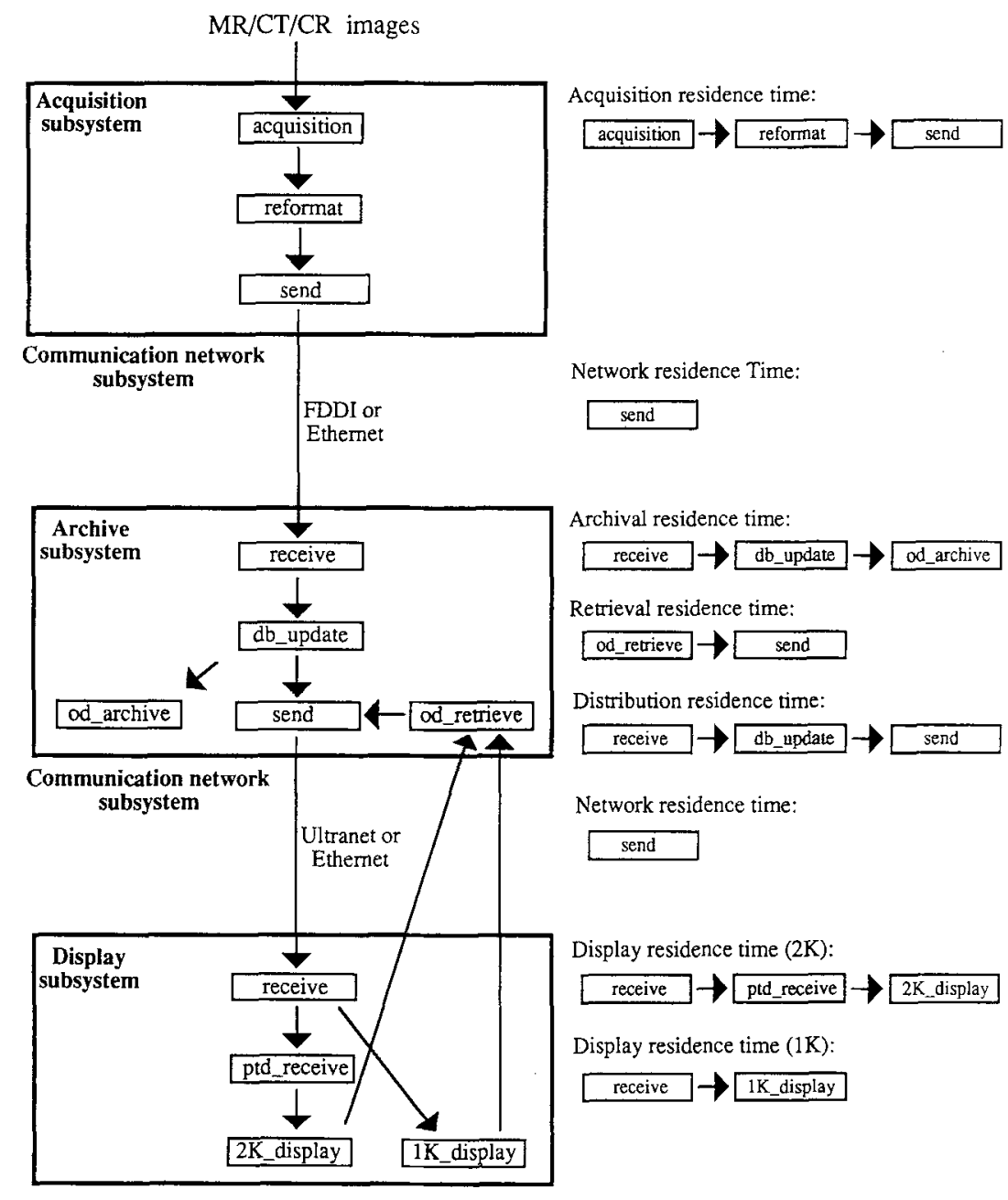

Fig 3. PACS subsystems and image residence times. 
the GE next-generation data interface, the ID/NET II. Genesis uses a Sun-3 computer for receiving images from the $\mathrm{CT}$ scanner. Because of hardware incompatibility, one of our four existing CT scanners does not use the Genesis data interface. Instead, this scanner uses an intermediate PC-AT to transmit images to the acquisition computer that uses General Electric's Datalink communication protocol.) The transfer of images to the PACS acquisition computers was controlled by the "push" operation on the scanners, which was generally done by the technologists when an examination was complete. This operator-oriented image transfer created a critical problem for our PACS concerning the timely delivery of current images to the display stations. Occasionally, an acquisition computer received no images for a certain period of time because the transfer of images had not been initiated from the scanner. On the other hand, the lack of information from the scanners indicating the completion of a scan also delayed the acquisition of a complete sequence of slice images (see explanation described in the previous section). Similar to the MR images, the acquisition time of a CT image sequence overlapped the reformat time of its individual slice images. The reformatted images were sent at an average size of 13.2 Mbytes to the PACS controllers via the FDDI or the Ethernet network.

CR image acquisition. Images from the PCR901 (Philips Medical Systems, Shelton, CT) was captured by our in-house hardware interface box, where they were truncated from their original 10 bits to 8 bits and sent to the acquisition computer at a data rate of $80 \mathrm{kbytes} / \mathrm{s}$ via a DR11-W data interface. Images from the two PCR-7000s (Philips Medical Systems) used the PIP (Philips interface processor) data interface to transmit images at full 10-bit pixel depth to the acquisition computers at a data rate of 236 kbytes/s via the Ethernet network. All CR images were reformatted to a size of $2 \mathrm{~K}$ and sent to the PACS controllers over the Ethernet network. Figure 4 shows the average acquisition residence times of the MR, CT, and CR images measured from the acquisition subsystem.

\section{Archival Residence Time}

Images in the PACS controllers were archived to the Kodak ADL 6800 optical disk
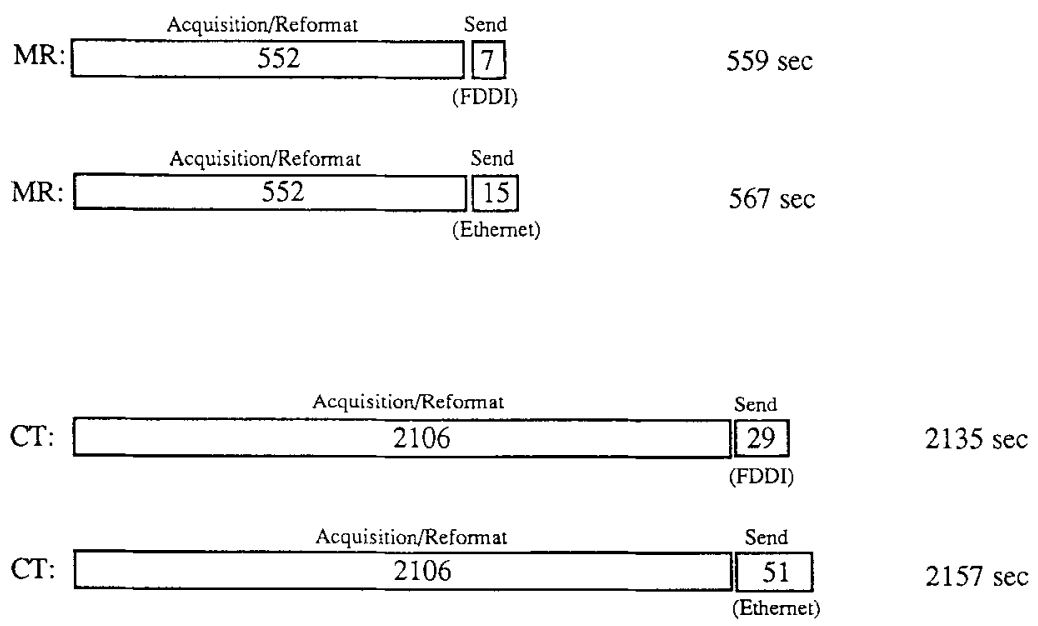

Fig 4. Acquisition subsystem throughput: acquisition residence time. Image size: MR, 1.96 Mbytes; CT, 13.2 Mbytes; CR, 4 Mbytes (PCR-901) and 8 Mbytes (PCR-7000).

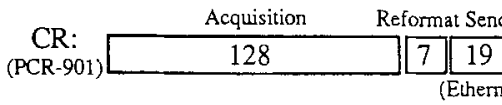

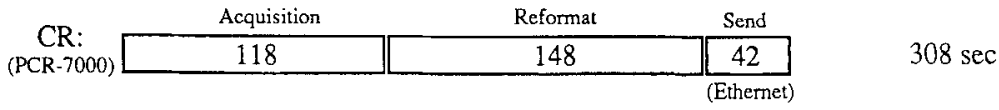


libraries at a data rate of $240 \mathrm{kbytes} / \mathrm{s}$ via an SCSI (small computer systems interface). (We allocated a smaller buffer size in the PACS controllers for the write/read operations of the Kodak optical libraries as a compromise to the large memory allocation for the high-speed UltraNet network. With their normal configuration, the optical libraries operate at data rates of 420 and $600 \mathrm{kbytes} / \mathrm{s}$ for write and read operations, respectively.) However, the archival residence time of an image was significantly affected by the job prioritizing mecha- nism used in the PACS controllers. Because of its low priority compared with the retrieve and "distribute" processes running on the archive subsystem, an archive process always compromised and waited if there was any retrieve or distribute job executing or pending. Figure 5A shows the archival residence time of images. (Archival residence time, retrieval residence time, and distribution residence time were measured on images in the archive subsystem according to an average image size of 7.5 Mbytes, regardless of modality. These residence times

Archival:

\begin{tabular}{|c|c|c|}
\hline Receive & Archive & \\
\hline 13 & 101 & $115 \mathrm{sec}$ \\
\hline (FDDi) & & \\
\hline & Archive & \\
\hline 24 & 101 & $126 \mathrm{sec}$ \\
\hline
\end{tabular}

(A)

Retrieval:

\begin{tabular}{|c|c|}
\hline \multicolumn{1}{|c}{ Retrieve } & Send \\
\hline 68 & 14 \\
\hline & (UleraNet) \\
\hline & \\
\hline Retrieve & Send \\
\hline 68 & 29 \\
\hline & $97 \mathrm{sec}$ \\
\hline &
\end{tabular}

(B)

Distribution:

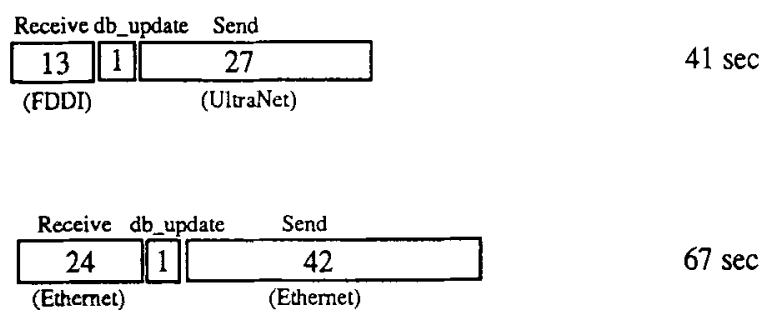


represented the required times for an image to be processed in the archive, retrieve, and distribute tasks.)

\section{Retrieval Residence Time}

Images were retrieved from the Kodak ADL 6800 optical disk libraries to the PACS controlIers at a data rate of $400 \mathrm{kbytes} / \mathrm{sec}$ via an SCSI interface. Among the three major processes carried on by the PACS controllers, handling retrieve requests always had the highest priority over the archive and distribute processes so that intended images for study comparison were retrieved and sent to the requested display stations immediately. During peak hospital hours, the two optical libraries, configured with four disk drives and two disk controllers, were able to handle multiple retrieval requests from different display stations with minimal delay. Figure 5B shows the retrieval residence time of images.

\section{Distribution Residence Time}

All images arriving in the PACS controllers were distributed immediately to their destination display stations before they were archived on optical disks. These images were sent to the $2 \mathrm{~K}$ stations via the fiber-optic UltraNet network at a data rate of $1.25 \mathrm{Mbytes} / \mathrm{s}$ or to the $1 \mathrm{~K}$ stations via the Ethernet network at 700 kbytes/s. Figure $5 \mathrm{C}$ shows the distribution residence time of images.

Display Residence Time ( $2 K$ )

Each $2 \mathrm{~K}$ display station was configured with a 2.6-Gbyte Concept-51 parallel transfer disk (ptd, Storage Concepts Inc, Irvine, CA). All arriving images in a $2 \mathrm{~K}$ display station were automatically transferred from the Sun host's magnetic disk to the ptd at a throughput of $1.07 \mathrm{Mbytes} / \mathrm{s}$. These images resided in the ptd and were displayed in the MegaScan portrait-mode monitors (AVP, Littleton, MA) at a data rate of 3.8 Mbytes/s. (We are implementing the direct transfer of images from the UltraNet network to the Concept-51 ptd's. Our attempt to bypass the Sun disks will minimize the immediate available time of the current images in the display stations.) Figure $6 \mathrm{~A}$ shows the display residence time of the $2 \mathrm{~K}$ (8-Mbyte) images.

\section{Display Residence Time (1K)}

Each $1 \mathrm{~K}$ display station was configured with a 1.3-Gbyte magnetic disk. Images were displayed in the portrait-mode monitors (Image Systems, Hopkins, MN) at a data rate of 1 Mbyte/s. Figure $6 \mathrm{~B}$ shows the display residence time of the $1 \mathrm{~K}$ (1-Mbyte) images.

\section{Communication Subsystem: Network Residence Time}

The residence time of an image in the multiple communication networks was measured as an overlapped residence time of the image in the acquisition, archive, and display subsystems (see above). The disk-to-disk transfer speed of the Ethernet, FDDI, and UltraNet networks are shown in Table 6.

\section{PACS Overall Throughput: Total Image \\ Residence Time}

The overall throughput of the PACS was determined by the total residence time of an

$2 \mathrm{~K}$ station:

\begin{tabular}{|c|c|}
\hline 11 & Receive \\
\hline (UltraNet) & 10 \\
\hline
\end{tabular}

24 seconds

Fig 6. Display subsystem throughput: display residence time. Image size: $2 \mathrm{~K}$ station, 8 Mbytes (2,048 × 2,048 $\times 2$ bytes); 1 K station, 1 Mbyte $\{1,024 \times$ $1,024 \times 1$ byte).
1K station:

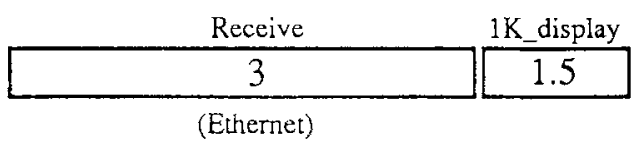

4.5 seconds 
Table 6. Disk-to-Disk Transfer Speed of Ethernet, FDDI, and UltraNet Networks (kbytes/s)

\begin{tabular}{lccc}
\hline & Ethernet & FDDI & UltraNet \\
\hline Point-to-point & 700 & 1,200 & 1,250 \\
PACS real time & 385 & 854 & 1,176 \\
\hline
\end{tabular}

NOTE: It is obvious that disk-to-disk transfer speed is limited by disk input/output. For memory-to-memory transfer, the FDDI and the UltraNet can reach $1.8 \mathrm{Mbytes} / \mathrm{s}$ and $5 \mathrm{Mbytes} / \mathrm{s}$, respectively.

image from its original source, a radiologic imaging device, to its ultimate destination, a display station or an optical disk. As an example, the total residence time of a CT image from acquisition to display was measured as the total of its acquisition residence time, distribution residence time, and display residence time (Table 7). In this example, a total of 2,205.5 seconds (or 36.8 minutes) were required for a 13.2Mbyte CT image to become available for review in a $2 \mathrm{~K}$ display station after an examination was complete.

\section{DISCUSSION}

Our implemented strategies including hardware redundancy, job prioritizing control, imageprefetch process, and open systems computing have resulted in a dramatic improvement in the overall performance of the PACS.

As an example of hardware redundancy, the dual archive subsystem ${ }^{11}$ (which consists of two optical libraries, the corresponding PACS controllers, and the multiple communication networks) benefits the required processing time on

\begin{tabular}{clc}
$\begin{array}{c}\text { Table 7. PACS Overall Throughput: } \\
\text { Time for a 13.2-Mbyte CT Image from }\end{array}$ & $\begin{array}{c}\text { Acquisition to Display } \\
\text { PACS }\end{array}$ & \multicolumn{1}{c}{$\begin{array}{c}\text { Required } \\
\text { Processing } \\
\text { Time (s) }\end{array}$} \\
\hline Subsystem & \multicolumn{1}{c}{ PACS Process } & 2,106 \\
\hline Acquisition & acquisition/reformat & 29 \\
Archive & send (over FDDI) & - \\
& receive & 1 \\
& db_update & $48 \dagger$ \\
Display & send (over UltraNet) & - \\
& receive & $16.5 \dagger$ \\
& ptd receive & $5 \dagger$ \\
Total processing time: & $2 \mathrm{~K}$ display & $2,205.5 \mathrm{~s}$ \\
& & (or $36.8 \mathrm{~min})$
\end{tabular}

tBased on the following required processing times: send $(7.5$ Mbytes): $27 \mathrm{~s}$ (Fig 5C); ptd_receive (8 Mbytes): $10 \mathrm{~s}$ (Fig 6A); and 2K-display (8 Mbytes): 3 s (Fig 6A). the images because data can be spread over these devices and consequently less competition for computer processing time among individual processes should be expected. On the other hand, using job prioritizing control allows urgent requests to be processed immediately. For example, a request from a display station in the archive subsystem to retrieve an image from an optical disk has the highest priority over any other processes running on the archive subsystem and is processed immediately. When the retrieval is complete, the image is queued for transmission with a priority higher than the rest of the images that have just arrived from the acquisition nodes and are waiting for transmission. During the retrieval, the archive process must be compromised and wait until the retrieval is complete. As an example, a 20-Mbyte image file takes 54 seconds to be retrieved from an optical library. However, if this image file is retrieved while another large image file is being archived to the same library, the retrieval time for the former image will be 96 seconds. In other words, the time delay of the retrieval without job prioritizing is 42 seconds. We have measured an average of $40 \%$ increase in overall performance of delivering individual images from the archive subsystem to the display subsystem during hospital hours ( 9 am to $5 \mathrm{pm}$ ). Moreover, to retrieve old images from the optical libraries for study comparison, the prefetch process in the display stations can relieve the workload of image retrieval during hospital hours and minimize the waiting time for the radiologists and referring clinicians to review these images. In addition, our PACS also takes advantage of the open architecture of Unix. Each Unix-based PACS computer operates independently with its own central processing unit and local storage, but they communicate with each other via the communication networks. This results in less competition for computer processing time and disk storage among individual PACS processes and each process can therefore be performed more efficiently and reliably.

The bottleneck in PACS performance is the low-speed data interface implemented in the radiologic imaging devices and the archive devices by the major manufacturers. For example, 
current system design of GE's MR and CT scanners has made it difficult for the PACS developers to provide prompt, on-line clinical service. Besides, a distributed PACS using a single-drive optical library to serve multiple display stations will be unable to meet the performance criteria. With its low-speed input/ output, this library can never promise a timely service if multiple retrieval requests are initiated from different display stations during clini- cal hours. Therefore, it is necessary for the manufacturers to speed up the image transmission from the imaging devices and the archive devices to improve the overall performance of the PACS.

\section{ACKNOWLEDGMENT}

The authors thank Dr Kathy Andriole, Dr Bruce K.T. Ho, and Dr Shyh-Liang Lou for their assistance in conducting the performance measurements.

\section{REFERENCES}

1. Huang HK, Lou SL, Cho PS, et al: Radiologic image communication methods. AJR Am J Roentgenol 155:183186,1990

2. Wong A, Stewart BK, Lou SL, et al: Multiple communication networks for a radiology PACS. Proc SPIE 1446:7380,1991

3. Stewart BK, Lou SL, Wong WK, et al: An ultra-fast network for radiologic image communication. AJR Am J Roentgenol 156:835-839, 1991

4. Huang HK, Kangarloo H, Cho PS, et al: Planning a totally digital radiology department. AJR Am J Roentgenol 154:635-639, 1990

5. Huang HK, Taira RK: Infrastructure design of a picture archiving and communication system. AJR Am J Roentgenol 158:743-749, 1992

6. Taira RK, Mankovich NJ, Boechat MI, et al: Design and implementation of a picture archiving and communica- tion system for pediatric radiology. AJR Am J Roentgenol 150:1117-1121, 1988

7. Cho PS, Huang HK, Tillisch J, et al: Clinical evaluation of a radiologic picture archiving and communication system for a coronary care unit. AJR Am J Roentgenol 151:823-827, 1988

8. Lou SL, Huang HK, Mankovich NJ, et al: A CT/ $\mathrm{MR} / \mathrm{US}$ picture archiving and communication system. Proc SPIE 1093:31-36, 1989

9. Weinberg WS, Loloyan M, Chan KK: On-line acquisition of CT and MRI studies from multiple scanners. Proc SPIE 1446:430-435, 1991

10. Ho BKT: Automatic acquisition interfaces for computed radiography, CT, MR, US, and laser scanner. Comput Med Imaging Graph 15:135-145, 1991

11. Wong AWK, Taira RK, Huang HK: Digital archive center: Implementation for a radiology department. AJR Am J Roentgenol 159, 1992 (in press) 